Monatsschr Kinderheilkd 2011 · 159:260-260

DOI 10.1007/s00112-010-2312-3

Online publiziert: 10. Dezember 2010

c) Springer-Verlag 2010

Redaktion

S. Wirth, Wuppertal

\author{
E. Herting \\ Klinik für Kinder- und Jugendmedizin, Universitätsklinikum \\ Schleswig-Holstein, Campus Lübeck
}

\title{
Intraossäre Infusion in der Kinder- und Jugendmedizin
}

In der in diesem Heft der Monatsschrift Kinderheilkunde abgedruckten Empfehlung der Arbeitskreise Notfallmedizin und des Arbeitskreises Kinderanästhesie der Deutschen Gesellschaft für Anästhesiologie und Intensivmedizin wird der aktuelle Stand zur Infusions- und medikamentösen Therapie über die so genannten Knochennadeln zusammengefasst.

Über einige Jahrzehnte war diese Form des Gefäßzuganges eher ein Geheimtipp speziell für den notfallmäßigen Einsatz im Säuglings- und Kleinkindesalter, in dem die venöse Punktion an üblicher Stelle entweder schwierig (Babyspeck, kurzer Hals) oder die Zugangsmöglichkeiten (z. B. über die Kopfgefäße) entweder unbekannt oder (außerhalb der Pädiatrie) ungeübt sind. Gerade im frühen Kindesalter erfordern die Notfallmaßnahmen aber eher ein primär auf die Luftwege ausgerichtetes Vorgehen, weswegen dem Autor diverse Notfallsituationen erinnerlich sind, in denen präklinisch und in der Klinik viel Zeit durch frustrane Venenpunktionsversuche bei Säuglingen vergeudet wurde, ohne dass an die Möglichkeit einer intraossären Infusion gedacht wurde.

\section{(D) Es wird immer noch zu selten an die Option der intraossären Infusion gedacht}

Diese Situation hat sich insofern geändert, als dass die intraossäre Infusion als Notfallmaßnahme in die nationalen und die internationalen Leitlinien aufgenommen wurde. Das Verfahren wird in entsprechenden Kursen jetzt sowohl von Notfall- medizinern als auch von Pädiatern in der Ausbildung an Modellen geübt. Auch in der Monatsschrift Kinderheilkunde wurde mehrfach über die Option des intraossären Gefäßzugangs berichtet und die neuen Applikationsmöglichkeiten (z. B. Bohrer) auch mittels Abbildungen vorgestellt. In der Klinik ist das Verfahren ebenfalls zur (kurzfristigen) Überbrückung geeignet; gerade bei älteren Kindern und Jugendlichen wird immer noch zu selten an diese Option zur Infusion gedacht.

Die zunehmende Verbreitung dieser Methode - auch in Kinderkliniken wird uns in Zukunft verdeutlichen, welche praktischen Probleme auch bei dieser Form des Gefäßzuganges auftreten und welche Nebenwirkungen zu erwarten sind.

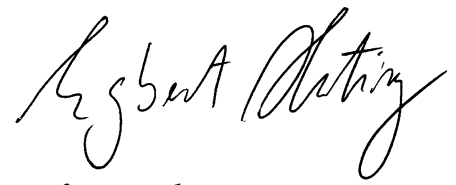

Prof. Dr. med. E. Herting

\section{Korrespondenzadresse}

Prof. Dr. E. Herting

Klinik für Kinder- und Jugendmedizin, Universitätsklinikum SchleswigHolstein, Campus Lübeck Ratzeburger Allee 160, 23538 Lübeck herting@paedia.ukl.mu-luebeck.de 